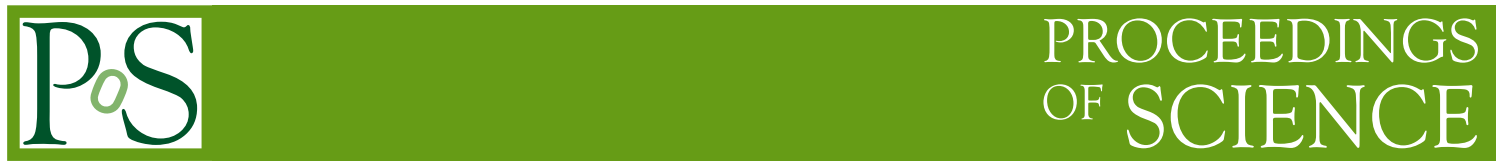

\title{
On the nature of the X-ray corona of black hole binaries in the hard state
}

\author{
Julien Malzac* \\ CESR (CNRS/Universite de Toulouse) \\ E-mail: malzacecesr.fr
}

\begin{abstract}
We discuss the nature of the X-ray emitting plasma of black hole binaries in the hard state. It is well known that the temperature and optical depth of the Comptonising electrons of the X-ray corona of black hole binaries can be measured using spectroscopy in the $1 \mathrm{keV}-1 \mathrm{MeV}$ energy band. We emphasize recent developments in the modeling of high energy radiation processes which allow us to constrain other important physical parameters of the corona, such as the strength of magnetic field, or the temperature of the ions. The results appear to challenge current accretion models. In particular, standard advection dominated accretion flow do not match the observed properties of bright hard state X-ray binaries such as Cygnus X-1 or GX 339-4. On the other hand we find that all the data would be consistent with a multi-zone magnetically dominated hot accretion flow model.
\end{abstract}

8th INTEGRAL Workshop ãThe Restless Gamma-ray Universeä

September 27-30 2010

Dublin Castle, Dublin, Ireland

\footnotetext{
${ }^{*}$ Speaker.
} 


\section{Observations and radiation model}

Black hole binaries are observed in two main X-ray spectral states, namely the Hard State (HS) and the Soft State (SS; see Done, Gierlinski and Kubota 2007). In the HS the high energy spectrum forms a power-law with photon index $\Gamma$ in the range 1.4-2 and a sharp high energy cut-of around $100 \mathrm{keV}$. These spectra are generally interpreted as thermal Comptonisation. Namely, optical, UV or soft X-ray photons are gradually up- scattered into the hard X-ray and gamma-ray domain through multiple Compton interactions with a population of hot thermal electrons of temperature $T_{\mathrm{e}} \sim 10^{9} \mathrm{~K}$ (Sunyaev \& Titarchuk 1980). Spectral fits with thermal Comptonization models allows to constrain the Thomson optical depth and temperature of the plasma. Typical parameters in the hard state are $\tau_{\mathrm{T}} \sim 1-3, k T_{\mathrm{e}} \sim 70-100 \mathrm{keV}$. It is worth stressing that in the HS the Thomson optical depth of the plasma is usually found to be larger than unity, which means that we are not dealing with an optically thin plasma. As we will see below, this has important consequences for dynamical models.

In addition a high energy excess above the cut-off energy is detected in the brightest sources (McConnell et al. 2002; Wardziński et al. 2002; Droulans et al. 2010, and see Fig. 1). This high energy excess is generally believed to be due to the presence of a population of energetic non-thermal electrons in the corona. In fact, the hard X-ray emission in all spectral states is well represented by Comptonisation by electrons with a hybrid thermal/non-thermal distribution (Poutanen \& Coppi 1998). In these models, the Comptonizing electron distribution is a Maxwellian at low energy with a power-law extension at high energy. The most common implementation of this hybrid thermal/non-thermal model is in the form of the 'EQPAIR' model (Coppi 1999). In this model the X-ray source is modelled as a spherical Comptonising cloud in which soft photons are uniformly and isotropically injected with a multicolour accretion disc spectrum. The Compton cloud is powered through two channels: 1) direct heating of the thermal electrons, 2) injection of high energy electron with a power-law distribution. The steady state particle and photon distribution in the cloud is then computed according to energy and electron/positron pair balance, as a function of the power input in the corona, as well as other parameters, such as the temperature of the soft seed photons, size of the emitting region or Thomson optical depth $\tau$ of the plasma. The calculation is performed in the one-zone isotropic approximation and radiative transfer is dealt with using an escape probability formalism.

EQPAIR was tested against many data sets. It appears to be the only model that is able to account for the spectra of all sources and in all spectral states observed so far. For instance, in 2004, INTEGRAL observed a spectral state transition of GX 339-4. This observation campaign allowed us to follow the evolution of the spectrum during the transition from HS to SS. Del Santo et al. (2008) fitted these spectra with the EQPAIR model. They found that the spectral evolution was consistent with a smooth transition from quasi-thermal to purely non-thermal Comptonisation.

More recently, Belmont, Malzac \& Marcowith (2008) developped the BELM code which, although similar to EQPAIR, improves upon EQPAIR in two ways: First it takes into account synchrotron emission and self-absorption. These effects can be important for the thermalization of the electrons in the hot plasma. Indeed, very fast emission and self-absorption of synchrotorn photons constitutes a very efficient way of exchanging energy between the leptons of the plasma. This coupling between high energy and lower energy particles acts as a very efficient thermalizer for the 
electron distribution. It will naturally form and keep the electron distribution Maxwellian (at least at low energies). This is the so-called synchrotron boiler effect first pointed out by Ghisellini et al. (1988). The synchrotron radiation is also Comptonized by the electrons and tends to cool down the electron distribution. The second improvement brought by BELM is that it takes into account the Coulomb interactions of the electrons with a population of thermal protons. This makes it possible to model the situation that occurs in 2-temperature accretion flow models where the electrons are heated essentially through Coulomb interactions with a population of hot protons which have a much higher temperature (see next section).

Malzac \& Belmont (2009, hereafter MB09) compare this model to the high energy spectra of Cynus X-1. This comparison allows us to constrain not only the usual Comptonisation parameters of the corona (the electron temperature, energy distribution and Thomson optical depth) but also other physical parameters, such as the magnetic field or the temperature of the ions. We find the magnetic field must be relatively low in the HS otherwise the synchrotron photons cool down the corona at temperatures below 50-100 keV that are typically observed. In fact if we compare the magnetic to radiation energy density in the HS, $U_{B} / U_{\mathrm{R}}<0.3$. This constraint on the magnetic field is a direct consequence of the presence in the HS spectra of a non-thermal high energy tail above the cut-of energy. Indeed this tail tightly constrains the number of non-thermal Comptonising electrons, which are also the main emitters of synchrotron radiation which in turn cools down the plasma. It turns out that the magnetic field must be significantly sub-equipartition in order to prevent the synchrotron radiation from cooling down the plasma below the observed range of electron temperatures. In the framework of one zone models the requirement of a low magnetic field appears extremely robust. Such requirement was already pointed out by Wardziński et al. $(2001,2002)$ who estimated analytically the synchrotron emission of the non-thermal electrons in (Cyg X-1 and GX339-4). We also note that Vurm and Poutanen (2009) independently developed a code similar to BELM and derived identical results regarding the magnetic field in the HS corona of Cygnus X-1 (Vurm \& Poutanen 2008; Poutanen \& Vurm 2009)

Using BELM, MB09 also investigated models in which most of the electron heating in the HS is provided by Coulomb collisions with a population of hot thermal protons. This also provides a very good description of the spectra of Cygnus X-1. However even in these models some level of non-thermal acceleration is required in order to reproduce the non-thermal $\mathrm{MeV}$ tails. In the $\mathrm{HS}$ the proton temperature appears significantly lower than what predicted by standard 2-temperature accretion flow solutions. We infer a proton temperature lower than $2 \mathrm{MeV}$ while typical ADAF models yield temperatures in the range $10-100 \mathrm{MeV}$. The low proton temperature that we infer is related to the relatively large Thomson optical depth $\left(\tau_{\mathrm{T}}>1\right)$ of the comptonising plasma. Indeed, since the plasma is not optically thin, the Coulomb coupling between ions and electrons is strong. A larger ion temperature would imply a larger luminosity than what is observed.

Recently Droulans et al. (2010) used BELM to model INTEGRAL spectra of GX339-4 during a bright hard state and found similar constraints on both the ion temperature and magnetic field. The modelling of the high energy spectra of black hole binaries therefore brings some interesting constraints on the magnetic field and ion temperature of the corona which, as will be discussed in the next section have important implications for dynamical models. 


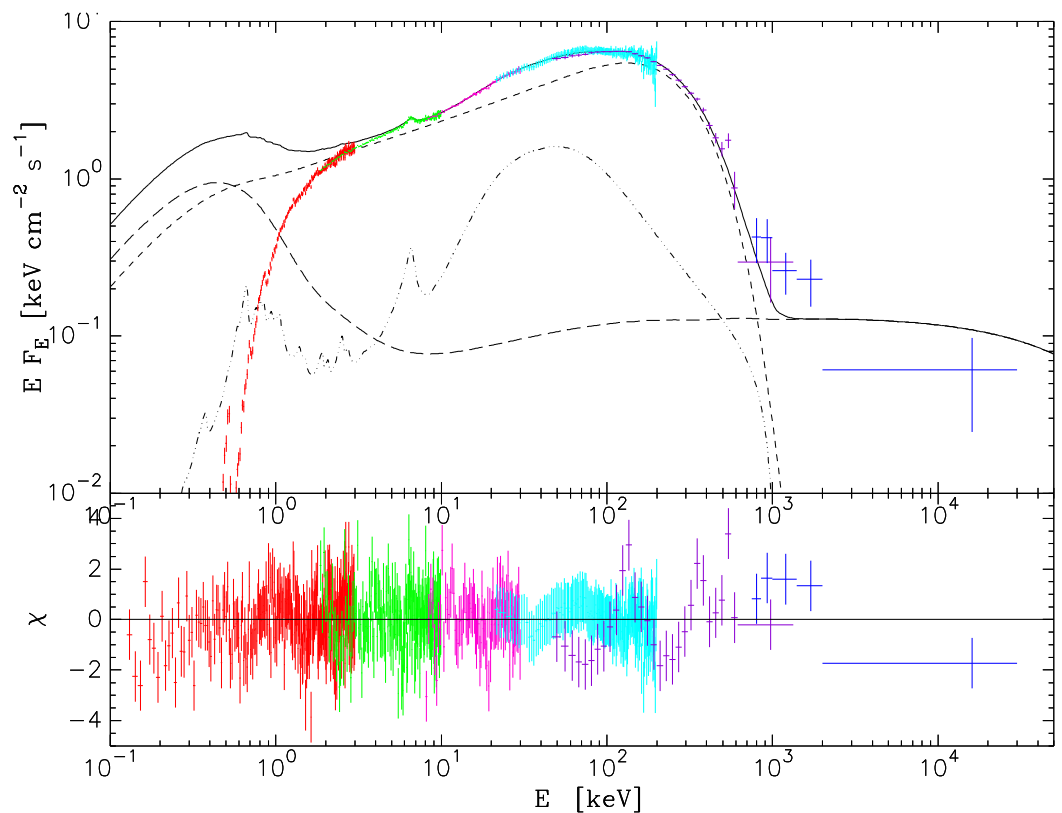

Figure 1: The joint Beppo-SAX/CGRO spectrum of Cygnus X-1 (McConnell et al. 2002) modeled with a multi-component models consisting of a pure thermal Comptonization region (short dashes) a hybrid thermal/non-thermal component (long dashes, including also the disc blackbody component) with heating of the Comptonizing electrons provided only through non-thermal acceleration and a relativistically smeared ionised reflection (3-dot-dash). The model is shown here unabsorbed. During the fit a fixed $N_{\mathrm{H}}=6.2 \times 10^{21} \mathrm{~cm}^{-2}$ column density of neutral absorbing material was assumed. The bottom panel shows the residuals of the absorbed best fit model.

\section{Physical models for the hard state}

There are currently several possible physical scenarios for the HS which are the subject of intense controversy. Among them the most popular is the truncated disc model (Done, Kubota and Gierliński 2007 and references therein). According to this picture, in the HS, the standard geometrically thin disc does not extend to the last stable orbit. Instead, it is truncated at distances ranging from a few hundred to a few thousand gravitational radii from the black hole (typically $1000-10000 \mathrm{~km}$ ). In the inner parts, the accretion flow takes the form of a hot geometrically thick, optically thin accretion flow. This hot flow is believed to correspond to hot two-temperature accretion flow solutions (such as the Advection Dominated Accretion Flow solution see Ichimaru 1977; Narayan and Yi 1994). In these hot acccretion flows the gravitational energy is converted through the process of viscous dissipation into the thermal energy of ions. The main coupling between the electrons and the ions is Coulomb interaction which is rather weak in the hot thin plasma. Since the cooling time of the ions is much longer than that of the electrons, the ion temperature is much higher than the electron temperature. However in the context of $\alpha$ discs (i.e. models in which the viscous heating is proportional to gas pressure) there is no hot flow solution with $\tau_{\mathrm{T}}>1$. If the optical depth is too large then the Coulomb coupling between ions and electrons is strong. The viscous heating cannot balance the cooling of ions through Coulomb collisions. The ion temperature drops and, as the flow is supported by the ion pressure, it collapses into a standard 
cold geometrically thin accretion disc. Therefore standard ADAF solutions cannot be applied to bright hard state sources such as Cygnus X-1 or GX 339-4. A possible solution was however suggested by Oda et al. (2010). These authors assume that the accretion flow in its innermost part is supported by the magnetic field and that the viscous heating is proportional to the total (i.e. gas plus magnetic presure). The strong magnetic field could be achieved both through diffusion from the external parts of the accretion flow and/or generated through dynamo processes in the hot flow itself. These authors show that hot flow solutions with $\tau_{\mathrm{T}}>1$ and ion and electron temperatures in the range observed in the HS can be achieved provided that the magnetic to gas pressure ratio is of order of a few. The requirement for such a high magnetic field is however in contradiction with the findings of MB09 and Poutanen \& Vurm 2009.

As an alternative, Beloborodov (1999) suggested that the HS emission could be produced in outflowing magnetically active regions similar to those of the soft state. Malzac, Beloborodov and Poutanen (2001) showed that this outflowing corona model could reproduce the HS spectra of Cygnus X-1 and other accreting black holes. This model however invoke magnetic dissipation as the source of the X-ray luminosity. This implies that the magnetic field must be larger than the upper limit found by MB09. Indeed, a weak magnetic field would require an unbelievably fast magnetic dissipation speed (see discussion in MB09).

Another alternative was proposed by several authors who suggested that the X-rays may actually be produced by the jet or its base (e.g. Georganopoulos et al. 2002, Kylafis et al. 2008, Markoff et al. 2005). It was shown however that, in Cygnus X-1 at least, the X-ray emission is very unlikely to be produced by the jet (see Malzac, Belmont \& Fabian 2009, Malzac \& Belmont 2010). The jet is nevertheless likely to be responsible for the gamma-ray emission that was detected by the MAGIC telescope (Albert et al. 2007; Malzac et al. 2008; Zdziarski et al. 2009). Finally we note that in all MHD jet models the magnetic field must be strong in order to be able to drive the accreting gas into a jet. This again appears in contradiction with the constraint for a low magnetic field discussed in the previous section.

\section{Toward a multizone model for the corona}

From the previous section it appears that none of the current physical scenarios is consistent with the constraint of weak magnetic field obtained from detailed modelling of the high energy spectrum. This constraint arises from the presence of a non-thermal component in the Comptonizing electron distribution wich is infered from the presence of a high energy excess in the spectra of bright HS sources. If the magnetic field is high then the synchrotron radiation from these electrons will tend to cool down the Mawellian component of the plasma out of the observed temperature range.

If the constraint for a low magnetic field is to be removed, we have to abandon the one-zone approximation and consider multi-zone models. Indeed, if the thermal and non-thermal electrons were located in different regions of the accretion flow, the synchrotron radiation from the nonthermal electrons would not affect the temperature of the thermal plasma. The magnetic field could then be large both in the region where the electrons are thermals and in the region with nonthermal electrons. For instance one could consider that the innermost part the accretion flow would be a hot magnetically dominated accretion flow producing the thermal Comptonisation compo- 
nent. In addition magnetic flares above and below the outer truncated accretion disc would produce a non-thermal Comptonisation spectrum similar to that observed in the soft state producing the non-thermal excess above the high energy cut-off. Along similar lines one could consider a magnetically powered outflowing corona in the central region producing the thermal comptonization while in the outer part the colder corona would be dominated by non-thermal comptonization. More complicated configurations can of course be envisioned.

In order to test the idea of a multi-zone corona, we attempted to fit the joint Beppo-SAX OSSE broadband (100 eV- $1 \mathrm{MeV})$ spectrum of Cygnus X-1 with a model consisting in two EQPAIR components one with pure thermal heating $\left(l_{\mathrm{nth}} / l_{\mathrm{h}}=0\right)$ the other with pure non-thermal acceleration. In addition we accounted for a ionised relativistically blurred reflection component (reflionx model in XSPEC). We found a good agreement with the data $\left(\chi^{2} / \mathrm{dof}=1.06\right)$. The result of the fit is shown in Fig. 1.

\section{Conclusion}

The nature of the X-ray corona of black hole binaries in the HS remains a mystery. In the best documented sources such as Cygnus X-1 or GX339-4 (which are also the brightests) none of the current physical scenarios (ADAF, outflowing corona or X-ray jet) really reproduce the full extent of the observed high energy spectral properties. In the context of hot accretion flows, magnetically dominated accretion flows appears to be a promising possibility that requires further investigation. Contrary to the results obtained from simple one zone comptonisation models, the magnetic field is likely to be strong in the corona (this is a requirement of most models). The magnetic field has important effects on the dynamic of the accretion flow, on the particle thermalisation and cooling and on the resulting emitted radiation. If the magnetic field is strong then the structure of the corona has to be more complex than previously envisioned: the radiation produced in different regions of the corona must have very different properties.

\section{References}

[1] Albert J., et al., 2007, ApJ, 665, L51

[2] Belmont R., Malzac J., Marcowith A., 2008, A\&A, 491, 617

[3] Beloborodov A. M., 1999, ApJ, 510, L123

[4] Coppi P. S., 1999, ASPC, 161, 375

[5] Del Santo M., Malzac J., Jourdain E., Belloni T., Ubertini P., 2008, MNRAS, 390, 227

[6] Droulans R., Belmont R., Malzac J., Jourdain E., 2010, ApJ, 717, 1022

[7] Done C., Gierliński M., Kubota A., 2007, A\&ARv, 15, 1

[8] Georganopoulos M., Aharonian F. A., Kirk J. G., 2002, A\&A, 388, L25

[9] Ghisellini G., Guilbert P. W., Svensson R., 1988, ApJ, 334, L5

[10] Ichimaru S., 1977, ApJ, 214, 840

[11] Kylafis N. D., Papadakis I. E., Reig P., Giannios D., Pooley G. G., 2008, A\&A, 489, 481 
[12] Malzac J., Beloborodov A. M., Poutanen J., 2001, MNRAS, 326, 417

[13] Malzac J., Lubiński P., Zdziarski A. A., Cadolle Bel M., Türler M., Laurent P., 2008, A\&A, 492, 527

[14] Malzac J., Belmont R., 2009, MNRAS, 392, 570

[15] Malzac J., Belmont R., Fabian A. C., 2009, MNRAS, 400, 1512

[16] Malzac J., Belmont R., 2010, IJMPD, 19, 769

[17] McConnell M. L., et al., 2002, ApJ, 572, 984

[18] Markoff S., Nowak M. A., Wilms J., 2005, ApJ, 635, 1203

[19] Oda H., Machida M., Nakamura K. E., Matsumoto R., 2010, ApJ, 712, 639

[20] Narayan R., Yi I., 1994, ApJ, 428, L13

[21] Poutanen J., Coppi P. S., 1998, PhST, 77, 57

[22] Poutanen J., Vurm I., 2009, ApJ, 690, L97

[23] Sunyaev R. A., Titarchuk L. G., 1980, A\&A, 86, 121

[24] Vurm I., Poutanen J., 2009, ApJ, 698, 293

[25] Vurm I., Poutanen J., 2008, IJMPD, 17, 1629

[26] Wardziński G., Zdziarski A. A., 2001, MNRAS, 325, 963

[27] Wardziński G., et al., MNRAS, 337, 829

[28] Zdziarski A. A., Malzac J., Bednarek W., 2009, MNRAS, 394, L41 